\title{
Modelling of transient radiation characteristics of a circular ultrasonic transducer
}

\author{
Horst Schwetlick ${ }^{\dagger}$ \\ Institut für Elektronik, Technische Universität Berlin, \\ Einsteinufer 19, D-1000 Berlin 10 F. R. G.
}

(Received 21 June 1988)

\begin{abstract}
A plain piston model is often not sufficient to describe the entire radiation characteristics of a ultrasonic transducer. In this report a transducer model is introduced, which bases on a decomposition of the transducer surface in concentric circular areas with uniform motion. A calibration procedure allows to compute the motion of these areas from the measured soundfield in a small number of spacial positions. The transient sound field at an arbitrary position is determined using these functions of motion.
\end{abstract}

PACS number: 43. 20. Px

Imaging procedures in ultrasonic testing are often based on a theory of planar or spherical wave propagation. Such sound fields can only approximately produced by regular piezoelectric ultrasonic transducers. In the approach presented here, the transducer is described by a wave theoretical model. This model can be implemented in the imaging process, in order to reduce errors caused by the directivity pattern and to enhance resolution.

The wavefield of a planar piston, moving in an infinite baffle, can serve as a rough model. The surface of the transducer is assumed to be rigid. The displacement in direction normal to the surface follows a prescribed time function. Figure 1 shows the geometry of this model. The time function of the sound pressure $p(t, r, z)$ at the spatial point $(r, z)$ in a infinite homogeneous medium is then given by the convolution

$$
p(t, r, z)=\rho_{0}\left[v^{\prime}(t) * h(t, r, z)\right]
$$

with $v^{\prime}(t)$ as the derivative of the membrane velocity and $\rho_{0}$ as the static density of the medium. The transfer function between a circular membrane and

$\uparrow$ Present address: Research Laboratory of Precision Machinery and Electronics, Tokyo Institute of Technology, 4259 Nagatsuta, Midori-ku, Yokohama, 227 Japan the spacial point $(r, z)$ is according to Stepanishen $(1971)^{1)}$ given by

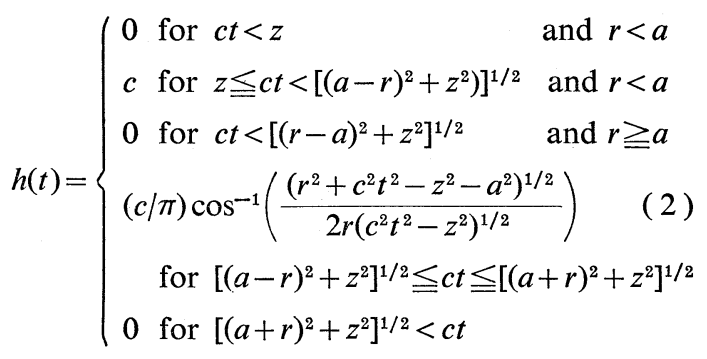

In this model all regions on the membrane are assumed to move with the same time function. In Harris $(1981)^{2)}$ a refined model is presented, where the velocity amplitude on the membrane is weighted for concentric regions.

In the Model presented here, the membrane is assumed to be synthesized on concentric regions moving with different time functions. Hence, the assumption of radial symmetry is maintained, but a nonuniform membrane motion is considered. The geometry of this model is shown in Fig. 2 and the sound pressure at the spatial point $(r, z)$ is given by

$$
p(t, r, z)=\sum_{i=1}^{N} v^{\prime} * h
$$




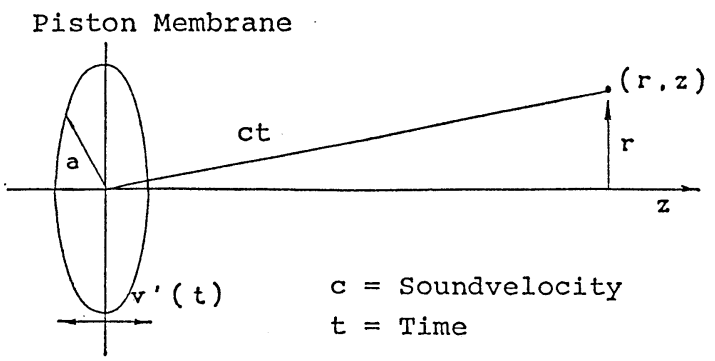

Fig. 1 Geometry for the soundfield of a piston membrane.

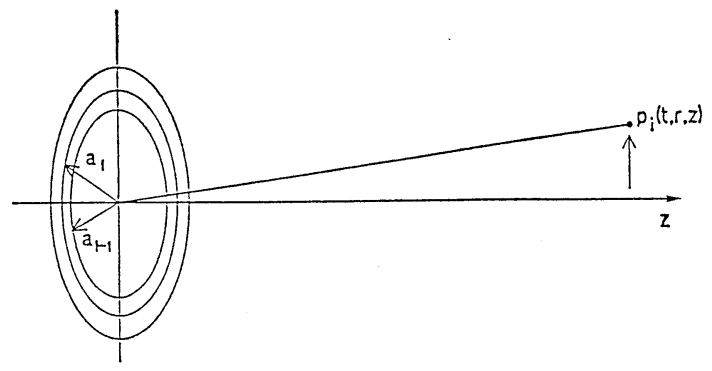

Fig. 2 Geometry for the ring model of the transducer.

i.e. by the contributions of a set of ring elements. The membrane velocity of a single ring element is represented by $v_{i}, h_{i}$ is the time domain transfer function from the $i$-th ring element to the chosen spatial point. The calculation of the transfer function of a piston membrane is given by Eq. (2), consequently the transfer function of a ring element is given by,

$$
h_{i}=h\left(t, r, z, a_{i}\right)-h\left(t, r, z, a_{i-1}\right)
$$

the difference of two piston membranes with different radius.

In order to characterize the transducer, the sound pressure is measured at $M$ different spatial points $p_{j}$. Fourier transform of Eq. (3) results in a set of equations for each frequency component $k$

$$
P_{j}(k)=\sum_{i=1}^{N} V_{i}(k) H_{i j}(k)
$$

where the transfer functions from the $i$-th ring element to the $j$-th spatial point is described by $H_{i j}$. After solving Eq. (5) for all frequency components for $v_{i}$, the actual time functions of the ring elements of the membrane are obtained by inverse Fourier transformation.

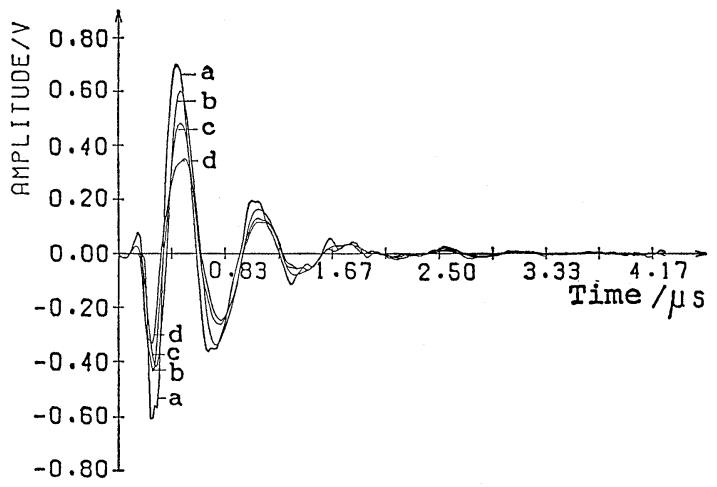

Fig. 3 Time iunction of the sound pressure in the medium. a) measured signal in a distance of $z=50 \mathrm{~mm}$ central to the membrane. The following time functions refer to the spatial point $r=2 \mathrm{~mm}$, $z=50 \mathrm{~mm}$. b) with the simple piston model calculated signal, c) with the ring model calculated signal, d) measured signal.

In the following practical example, this characterization was applied to a sending planar piezotransducer with $6.3 \mathrm{~mm}$ diameter. The soundfield in water was measured by a miniature hydrophone. In the chosen model, the membrane was synthesized by two concentric areas, a circular area with $4.8 \mathrm{~mm}$ diameter and a ring element with $4.8 \mathrm{~mm}$ inner diameter and $6.3 \mathrm{~mm}$ outer diameter. From measurement at $M=3$ points in the sound field, the characterizing time functions of the membrane velocity were determined by inverting Eq. (5). Figure 3 shows the comparison of the sound field at a spatial point between the measured time function, the synthesized time function with a simple piston model and the synthesized time function with a piston model. It can be seen that even this simple model already leads to an improvement in the description of the sound field. Prior to implementation in an imaging procedure, model calculations and experiments in order to find the optimal separation of the membrane for the particular transducer is necessary. The extension of this model to focus transducers is subject of our current research.

\section{ACKNOWLEDGEMENTS}

The Author feels deeply indebted to his late teacher W. Kessel from the Technical University of 


\section{H. SCHWETLICK: CHARACTERISTICS OF A CIRCULAR TRANSDUCER}

Berlin. He is very thankful to M. Ueda, Tokyo Institute of Technology, for introducing him to his work on transducer characterization. The support of the Deutsche Forschungsgemeinschaft, the Japan Society for the Promotion of Science, and the Alexander von Humboldt-Stiftung is gratefully acknowledged.

\section{REFERENCES}

1) P. R. Stepanishen, "Transient radiation from pistons in an infinite planar baffle," J. Acoust. Soc. Am. 49, 1629-1638 (1971).

2) R. Harris, "Transient field of a baffled planar piston having an arbitrary vibration amplitude distribution," J. Acoust. Soc. Am. 70, 186-204 (1981). 